\title{
A KATO PERTURBATION-TYPE RESULT FOR OPEN LINEAR RELATIONS IN NORMED SPACES
}

\author{
DANA GHEORGHE
}

\author{
(Received 21 April 2008)
}

\begin{abstract}
Using some techniques of perturbation theory for Banach space complexes, we obtain necessary and sufficient conditions for the stability of the topological index of an open linear relation under small (with respect to the gap topology) perturbations with linear relations.
\end{abstract}

2000 Mathematics subject classification: primary 47A06; secondary 47A53.

Keywords and phrases: linear relations, topological index, Banach space complexes.

\section{Introduction and the main result}

Several papers (see, for example, [2-5, 9] and the references therein) have been devoted to the study of the stability of the index of a linear relation under perturbations with continuous linear relations having small norm. The aim of this paper is to complete or extend some of these results. We prove a Kato-type result concerning the behavior of the topological index of an open linear relation between normed spaces under small perturbations with linear relations. The smallness is measured in the gap topology (see [12]) and it seems that, even in the context of linear operators, our main result is new because the perturbation is not necessarily continuous.

The paper is organized as follows. In Section 2 we introduce Banach space complexes and state our main tool, the Albrecht-Vasilescu perturbation theorem for complexes of Banach spaces [1, Theorem II.1.6]. We also prove some auxiliary results concerning the duality theory in normed spaces. Section 3 contains the proof of our main result.

We follow the notation and terminology of the monograph [9]. Let $X$ and $Y$ be two normed spaces. We denote by $\mathcal{B}(X, Y)$ the normed space of continuous linear operators acting from $X$ into $Y$. If $T \in \mathcal{B}(X, Y)$, then $R(T)$ denotes the range of $T$, $N(T)$ denotes the kernel of $T$ and $T^{\prime} \in \mathcal{B}\left(Y^{\prime}, X^{\prime}\right)$ denotes the adjoint of $T$.

Following Arens [7], a linear subspace $Z$ of $X \times Y$ is called a linear relation between $X$ and $Y$. If $Z$ is a linear relation, then we associate with it the linear subspaces

$$
D(Z)=\{x \in X ; \exists y \in Y,(x, y) \in Z\}
$$

(c) 2009 Australian Mathematical Society 0004-9727/2009 \$16.00 
called the domain of $Z$,

$$
R(Z)=\{y \in Y ; \exists x \in X,(x, y) \in Z\}
$$

called the range of $Z, N(Z)=\{x \in D(Z) ;(x, 0) \in Z\}$ called the kernel of $Z$, and

$$
M(Z)=\{y \in R(Z) ;(0, y) \in Z\}
$$

its multivalued part. The set $\{y \in Y ;(x, y) \in Z\}$ is denoted by $Z(x)$. A linear relation $Z$ can also be identified with the graph of the multivalued linear operator $T: D(Z) \rightarrow \mathcal{P}(Y) \backslash\{\emptyset\}$ defined by $T x=Z(x)(x \in D(Z))$, where $\mathcal{P}(Y)$ denotes the family of all subsets of $Y$.

A linear relation $Z$ is said to be open if whenever $U$ is a neighbourhood in $D(Z)$, the image $Z(U):=\bigcup_{x \in U} Z(x)$ is a neighbourhood in $R(Z)$. Note that $Z$ is open if and only if there exists $\rho>0$ such that

$$
\rho B_{Y} \cap R(Z) \subset Z\left(B_{X} \cap D(Z)\right),
$$

where $B_{X}$ and $B_{Y}$ denote the closed unit balls of $X$ and $Y$ respectively (see [9, Propositions II.2.4 and II.3.2 (b)]). Assume that the linear relation $Z$ satisfies

$$
\operatorname{dim}(N(Z))<\infty \quad \text { or } \quad \operatorname{codim}(\overline{R(Z)})<\infty
$$

Then the quantity

$$
\overline{\operatorname{ind}}(Z)=\operatorname{dim}(N(Z))-\operatorname{codim}(\overline{R(Z)})
$$

is called the topological index of $Z$ (see [9, 11]).

The importance of the investigation of linear relations is demonstrated by the necessity of taking adjoints of operators with a nondense domain used in applications to the theory of generalized differential equations [8], or by the need to consider the inverse of certain linear operators, used, for example, in the study of some Cauchy problems associated with parabolic type equations in Banach spaces [10].

In order to state our main result we need to introduce a notion of 'distance' between two closed subspaces of a normed space. According to [6, Definition I.6.6] (see also [12]), if $M$ and $N$ are closed subspaces of a normed space $X$, then the gap between $M$ and $N$ is defined by

$$
\widehat{\delta}(M, N)=\max \{\delta(M, N), \delta(N, M)\},
$$

where

$$
\delta(M, N)=\sup _{x \in M,\|x\| \leq 1} \inf _{y \in N}\|x-y\|
$$

The main result of this paper is as follows. 
THEOREM 1. Let $X, Y$ be normed spaces and $Z_{1} \subset X \times Y$ be an open linear relation satisfying

$$
\operatorname{dim}\left(N\left(Z_{1}\right)\right)<\infty \text { and } \operatorname{codim}\left(\overline{R\left(Z_{1}\right)}\right)<\infty .
$$

There exists $\varepsilon>0$ such that for any linear relation $Z_{2} \subset X \times Y$ with

$$
M\left(Z_{1}\right)=M\left(Z_{2}\right) \text { and } \widehat{\delta}\left(\overline{Z_{1}}, \overline{Z_{2}}\right)<\varepsilon,
$$

the inequalities

$$
\operatorname{dim}\left(N\left(Z_{2}\right)\right) \leq \operatorname{dim}\left(N\left(Z_{1}\right)\right), \quad \operatorname{codim}\left(\overline{R\left(Z_{2}\right)}\right) \leq \operatorname{codim}\left(\overline{R\left(Z_{1}\right)}\right),
$$

and

$$
\overline{\operatorname{ind}}\left(Z_{2}\right) \leq \overline{\operatorname{ind}}\left(Z_{1}\right)
$$

hold. Moreover, $\overline{\operatorname{ind}}\left(Z_{2}\right)=\overline{\operatorname{ind}}\left(Z_{1}\right)$ if and only if $Z_{2}$ is open.

Results of this type were initiated by Kato [12, Ch. IV, Sections 4 and 5]. It is interesting to note that in the preceding theorem the linear relation $Z_{2}$ is not necessarily a perturbation of $Z_{1}$ with a continuous linear relation. A very special case of Theorem 1 is given in the following.

Example 2. Let $X, Y$ be normed spaces and $T_{1}: D\left(T_{1}\right) \rightarrow Y$ be a linear operator. Assume that $T_{1}$ is bijective and open, and consider a linear operator $T_{2}: D\left(T_{2}\right) \rightarrow Y$ such that $\widehat{\delta}\left(\overline{G\left(T_{1}\right)}, \overline{G\left(T_{2}\right)}\right)$ is sufficiently small. Using Theorem 1, it follows that $T_{2}$ is injective, has a dense range, is open and its topological index is zero. In particular, if the range of $T_{2}$ is closed, then $T_{2}$ is bijective with a continuous inverse. Hence, if the linear equation $T_{1} x=y$ has a unique solution $x$, for all $y \in Y$, depending continuously upon the initial data $y$, then the same holds true for the linear equation $T_{2} x=y$ provided that $T_{2}$ has a closed range and the gap between the closures of the graphs of $T_{1}$ and $T_{2}$ is sufficiently small.

The following corollary is a consequence of Theorem 1 .

Corollary 3. Let $X, Y$ be two normed spaces and $Z \subset X \times Y$ be an open linear relation such that

$$
\operatorname{dim}(N(Z))<\infty \text { and } \operatorname{codim}(\overline{R(Z)})<\infty .
$$

There exists $\varepsilon>0$ such that for every $A \in \mathcal{B}(D(A), Y)$ with $D(Z) \subset D(A)$ and $\|A\| \leq \varepsilon$, the inequalities

$$
\operatorname{dim}(N(Z+A)) \leq \operatorname{dim}(N(Z)), \quad \operatorname{codim}(\overline{R(Z+A)}) \leq \operatorname{codim}(\overline{R(Z)}),
$$

and

$$
\overline{\operatorname{ind}}(Z+A) \leq \overline{\operatorname{ind}}(Z)
$$

hold. Moreover, $\overline{\operatorname{ind}}(Z+A)=\overline{\operatorname{ind}}(Z)$ if and only if $Z+A$ is open. 
In the preceding corollary, the linear relation $Z+A$ is defined by

$$
Z+A:=\{(x, y+A x) ;(x, y) \in Z\}
$$

that is, $(Z+A)(x)=Z(x)+A x$ for all $x \in D(Z)$.

A result similar to Corollary 3 was obtained by Cross (see [9, Theorem V.15.6]), so Theorem 1 completes the main result concerning the stability of the index given in [9].

\section{Main tools and auxiliary results}

Our main tool comes from the theory of complexes of Banach spaces (see [6]) and will be stated in this section together with some well-known duality results necessary for the proof of our main result.

Let $\mathcal{X}, \mathcal{Y}$ be two normed spaces. A quotient morphism is a linear mapping $T: X / X_{0} \rightarrow Y / Y_{0}$ where $X_{0} \subset X \subset \mathcal{X}, Y_{0} \subset Y \subset \mathcal{Y}$ are linear subspaces. In $[13,14]$ Vasilescu introduced an important subspace of $X \times Y$ associated with $T$, namely the lifted graph of $T$ denoted by $G_{0}(T)$ and defined by

$$
G_{0}(T):=\left\{(x, y) \in X \times Y ; T\left(x+X_{0}\right)=y+Y_{0}\right\} .
$$

Let $X_{1}, X_{2}, \ldots, X_{n}$ be normed spaces, $\alpha_{i} \in \mathcal{B}\left(X_{i}, X_{i+1}\right)(i=1,2, \ldots, n-1)$ and $\alpha_{0}:\{0\} \rightarrow X_{1}, \alpha_{n}: X_{n} \rightarrow\{0\}$ be the zero operators (which will be omitted in what follows). The sequence

$$
0 \rightarrow X_{1} \stackrel{\alpha_{1}}{\longrightarrow} X_{2} \stackrel{\alpha_{2}}{\longrightarrow} \cdots \stackrel{\alpha_{n-2}}{\longrightarrow} X_{n-1} \stackrel{\alpha_{n-1}}{\longrightarrow} X_{n} \rightarrow 0
$$

is called a complex of normed spaces if

$$
R\left(\alpha_{i}\right) \subset N\left(\alpha_{i+1}\right) \quad(i=1, \ldots, n-2) .
$$

The complex $\alpha$ is said to be Fredholm if the quantities

$$
\operatorname{dim}\left(N\left(\alpha_{i+1}\right) / R\left(\alpha_{i}\right)\right) \quad(i=0, \ldots, n-1)
$$

are finite. In this case the index (or the Euler characteristic) of $\alpha$ is defined by

$$
\operatorname{ind}(\alpha):=\sum_{i=0}^{n-1}(-1)^{i} \operatorname{dim}\left(N\left(\alpha_{i+1}\right) / R\left(\alpha_{i}\right)\right) .
$$

The product of $n$ normed spaces $X_{i}(i=1,2, \ldots, n)$ will be endowed with the norm

$$
\left\|\left(x_{1}, x_{2}, \ldots, x_{n}\right)\right\|=\sum_{i=1}^{n}\left\|x_{i}\right\| .
$$

Albrecht and Vasilescu in [1, Theorem 1.6] (see also [6, Theorem 2.7]) obtain, roughly speaking, a stability result for the index of a complex of quotient Banach spaces under 'small' perturbations. Their result is our main tool and is as follows. 
Theorem 4. Let $\mathcal{X}$ be a Banach space and $X_{0}^{i} \subset X^{i} \subset \mathcal{X}(i=1,2, \ldots, n)$ be closed subspaces of $\mathcal{X}$. Assume that the sequence of quotient Banach spaces

$$
0 \rightarrow X^{1} / X_{0}^{1} \stackrel{\sigma_{1}}{\rightarrow} X^{2} / X_{0}^{2} \stackrel{\sigma_{2}}{\rightarrow} \cdots \stackrel{\sigma_{n-2}}{\longrightarrow} X^{n-1} / X_{0}^{n-1} \stackrel{\sigma_{n-1}}{\longrightarrow} X^{n} / X_{0}^{n} \rightarrow 0
$$

is a Fredholm complex. There exists $\delta_{\sigma}>0$ such that every complex of quotient Banach spaces

$$
0 \rightarrow \widetilde{X}^{1} / \widetilde{X}_{0}^{1} \stackrel{\widetilde{\sigma}_{1}}{\longrightarrow} \widetilde{X}^{2} / \widetilde{X}_{0}^{2} \stackrel{\widetilde{\sigma}_{2}}{\longrightarrow} \cdots \stackrel{\widetilde{\sigma}_{n-2}}{\longrightarrow} \widetilde{X}^{n-1} / \widetilde{X}_{0}^{n-1} \stackrel{\widetilde{\sigma}_{n-1}}{\longrightarrow} \widetilde{X}^{n} / \widetilde{X}_{0}^{n} \rightarrow 0
$$

which satisfies $\widehat{\delta}_{0}(\sigma, \widetilde{\sigma})<\delta_{\sigma}$ is Fredholm and $\operatorname{ind}(\sigma)=\operatorname{ind}(\widetilde{\sigma})$. Moreover,

$$
\operatorname{dim}\left(N\left(\widetilde{\sigma}_{i}\right) / R\left(\widetilde{\sigma}_{i-1}\right)\right) \leq \operatorname{dim}\left(N\left(\sigma_{i}\right) / R\left(\sigma_{i-1}\right)\right) \quad(i=1,2, \ldots, n) .
$$

In Theorem 4 the quantity $\widehat{\delta}_{0}(\sigma, \widetilde{\sigma})$ measures the 'distance' between the complexes $\sigma$ and $\widetilde{\sigma}$ and is defined as

$$
\widehat{\delta}_{0}(\sigma, \widetilde{\sigma}):=\sup _{i=0, \ldots, n} \widehat{\delta}\left(G_{0}\left(\sigma_{i}\right), G_{0}\left(\widetilde{\sigma}_{i}\right)\right)
$$

In the next lemma we introduce some useful isometric isomorphisms.

LEMMA 5. Let $X$ be a normed space and $M, N$ be linear subspaces of $X$ such that $N$ is closed in $X$ and $N \subset M$.

(i) The mapping

$$
\varphi: X^{\prime} / M^{\perp} \rightarrow M^{\prime}, \quad \varphi\left(x^{\prime}+M^{\perp}\right)=x^{\prime} \mid M
$$

is an isometric isomorphism. We recall that $M^{\perp}:=\left\{x^{\prime} \in X^{\prime} ; x^{\prime} \mid M=0\right\}$.

(ii) If $\pi: M \rightarrow M / N$ is the canonical surjection, then the mapping

$$
\psi:(M / N)^{\prime} \rightarrow N^{\perp} / M^{\perp}, \quad \psi=\varphi^{-1} \circ \pi^{\prime}
$$

is an isometric isomorphism.

(iii) If in addition $M$ is closed in $X$, then $\delta(M, N)=\delta\left(N^{\perp}, M^{\perp}\right)$.

PROOF. For the proof of (i) see [9, Proposition III.1.8 (a)] and for the proof of (ii) in the case $M=X$ see [9, Proposition III.1.8 (b)]. For the proofs of (ii) and (iii) in the Banach space context see [6, Propositions 8.5 and 8.10].

Remark 6. Let $X$ be a normed space and $M, N$ be subspaces of $X$ such that $N$ is closed in $X$ and $N \subset M$. From the above lemma it follows that

$$
\operatorname{dim}(M / N)=\operatorname{dim}\left((M / N)^{\prime}\right)=\operatorname{dim}\left(N^{\perp} / M^{\perp}\right) .
$$

In particular, if $N$ is a linear subspace of $X$, then

$$
\operatorname{dim}(X / \bar{N})=\operatorname{dim}\left(N^{\perp}\right) .
$$


The following lemma gives some duality results and is essential for the proof of the main result of this paper.

Lemma 7. Let $X, Y$ be two normed spaces and $T \in \mathcal{B}(X, Y)$. Then, we have the following results.

(i) $\overline{R\left(T^{\prime}\right)} \subset N(T)^{\perp}$ and $R(T)^{\perp}=N\left(T^{\prime}\right)$.

(ii) The operator $T$ is open if and only if $R\left(T^{\prime}\right)=N(T)^{\perp}$.

(iii) $\operatorname{codim}(\overline{R(T)})=\operatorname{dim}\left(N\left(T^{\prime}\right)\right)$ and $\operatorname{dim}(N(T)) \leq \operatorname{codim}\left(\overline{R\left(T^{\prime}\right)}\right)$.

(iv) If $T$ is open then $R\left(T^{\prime}\right)$ is closed and $\operatorname{dim}(N(T))=\operatorname{codim}\left(R\left(T^{\prime}\right)\right)$. Moreover, if $R\left(T^{\prime}\right)$ is closed and $\operatorname{dim}(N(T))=\operatorname{codim}\left(R\left(T^{\prime}\right)\right)<\infty$, then $T$ is open.

Proof. For the proofs of (i) and (ii) see, for example, [9, Propositions III.1.4 (a) and III.4.6 (b)] (in the more general context of linear relations).

(iii) Using Remark 6 and (i), it follows that

$$
\operatorname{codim}(\overline{R(T)})=\operatorname{dim}(Y / \overline{R(T)})=\operatorname{dim}\left(R(T)^{\perp}\right)=\operatorname{dim}\left(N\left(T^{\prime}\right)\right) .
$$

Using Remark 6 and Lemma 5, we infer that

$$
\operatorname{dim}(N(T))=\operatorname{dim}\left(X^{\prime} / N(T)^{\perp}\right) .
$$

On the other hand, because $\overline{R\left(T^{\prime}\right)} \subset N(T)^{\perp} \subset X^{\prime}$, it follows that

$$
X^{\prime} / N(T)^{\perp} \simeq\left[X^{\prime} / \overline{R\left(T^{\prime}\right)}\right] /\left[N(T)^{\perp} / \overline{R\left(T^{\prime}\right)}\right],
$$

which implies that

$$
\operatorname{dim}\left(X^{\prime} / N(T)^{\perp}\right)+\operatorname{dim}\left(N(T)^{\perp} / \overline{R\left(T^{\prime}\right)}\right)=\operatorname{dim}\left(X^{\prime} / \overline{R\left(T^{\prime}\right)}\right) .
$$

Consequently, from (4) and (5) it follows that

$$
\operatorname{dim}(N(T)) \leq \operatorname{dim}\left(X^{\prime} / \overline{R\left(T^{\prime}\right)}\right) .
$$

(iv) Assume that $T$ is open. Then, using (ii), (4) and (5), it follows that $R\left(T^{\prime}\right)$ is closed and

$$
\operatorname{dim}(N(T))=\operatorname{dim}\left(X^{\prime} / N(T)^{\perp}\right)=\operatorname{dim}\left(X^{\prime} / R\left(T^{\prime}\right)\right) .
$$

Now, assume that $R\left(T^{\prime}\right)$ is closed and $\operatorname{dim}(N(T))=\operatorname{codim}\left(R\left(T^{\prime}\right)\right)<\infty$. Then, using (4) and (5), we deduce that $\operatorname{dim}\left(N(T)^{\perp} / R\left(T^{\prime}\right)\right)=0$. This implies that $N(T)^{\perp}=R\left(T^{\prime}\right)$ and, using (ii), it follows that $T$ is open.

\section{Proof of the main result}

The next lemma is the key ingredient in the proof of the main result of this paper. If $Z$ is a linear relation, then we associate with it a complex of normed spaces which is strongly related to $Z$. 
Lemma 8. Let $X, Y$ be two normed spaces and let $Z \subset X \times Y$ be a linear relation. Consider the short sequence

$$
0 \rightarrow M(Z) \stackrel{i_{Z}}{\longrightarrow} Z \times M(Z) \stackrel{j_{Z}}{\longrightarrow} Y \rightarrow 0
$$

with

$$
\begin{aligned}
i_{Z}\left(y_{0}\right) & =\left(\left(0, y_{0}\right), y_{0}\right), \quad \forall y_{0} \in M(Z), \\
j_{Z}\left((x, y), y_{0}\right) & =y-y_{0}, \quad \forall\left((x, y), y_{0}\right) \in Z \times M(Z) .
\end{aligned}
$$

Then (6) is a complex of normed spaces satisfying

$$
N\left(i_{Z}\right)=\{0\}, \quad R\left(j_{Z}\right)=R(Z)
$$

and

$$
\operatorname{dim}\left(N\left(j_{Z}\right) / R\left(i_{Z}\right)\right)=\operatorname{dim}(N(Z)) .
$$

Moreover, the relation $Z$ is open if and only if the operator $j_{Z}$ is open. Finally, $R\left(i_{Z}\right)$ is closed in $Z \times M(Z)$.

PROOF. A simple calculation shows that

$$
N\left(j_{Z}\right)=\left\{\left(\left(x, y_{0}\right), y_{0}\right) ; x \in N(Z), y_{0} \in M(Z)\right\} .
$$

Indeed, consider $x \in N(Z)$ and $y_{0} \in M(Z)$. It follows that $(x, 0) \in Z$ and $\left(0, y_{0}\right) \in Z$. This implies that $\left(x, y_{0}\right) \in Z$, so $\left(\left(x, y_{0}\right), y_{0}\right) \in Z \times M(Z)$ and $j_{Z}\left(\left(x, y_{0}\right), y_{0}\right)=0$. That is, $\left(\left(x, y_{0}\right), y_{0}\right) \in N\left(j_{Z}\right)$. Conversely, assume that $\left((x, y), y_{0}\right) \in N\left(j_{Z}\right)$. It follows that $y=y_{0}$ and, using $\left(x, y-y_{0}\right) \in Z$, we deduce that $(x, 0) \in Z$. That is, $x \in N(Z)$ and the claim is proved.

It follows that $R\left(i_{Z}\right) \subset N\left(j_{Z}\right)$, so (6) is a complex (of vector spaces). Consider the linear operator

$$
\varphi: N\left(j_{Z}\right) \rightarrow X, \quad \varphi\left(\left(x, y_{0}\right), y_{0}\right)=x
$$

It follows that

$$
D(\varphi)=N\left(j_{Z}\right), \quad N(\varphi)=R\left(i_{Z}\right), \quad R(\varphi)=N(Z),
$$

which together with

$$
\operatorname{dim}(D(\varphi) / N(\varphi))=\operatorname{dim}(R(\varphi))
$$

imply that

$$
\operatorname{dim}\left(N\left(j_{Z}\right) / R\left(i_{Z}\right)\right)=\operatorname{dim}(N(Z)) .
$$

The claims concerning $N\left(i_{Z}\right)$ and $R\left(j_{Z}\right)$ are now obvious. 
Now let us prove the continuity of $i_{Z}$ and $j_{Z}$. It follows from

$$
\left\|i_{Z}\left(y_{0}\right)\right\|=\left\|\left(\left(0, y_{0}\right), y_{0}\right)\right\|=2\left\|y_{0}\right\|, \quad \forall y_{0} \in M(Z),
$$

and

$$
\begin{aligned}
\left\|j_{Z}\left((x, y), y_{0}\right)\right\| & \leq\|y\|+\left\|y_{0}\right\| \\
& \leq\left\|\left((x, y), y_{0}\right)\right\|, \quad \forall\left((x, y), y_{0}\right) \in Z \times M(Z),
\end{aligned}
$$

that $i_{Z}$ and $j_{Z}$ are continuous, hence the short sequence (6) is a complex of normed spaces.

Assume that $Z$ is open. This implies that there exists a constant $\rho>0$ such that

$$
\rho B_{Y} \cap R(Z) \subset Z\left(B_{X} \cap D(Z)\right) .
$$

Consider $y \in R\left(j_{Z}\right)=R(Z)$ such that $y \neq 0$. This implies that $\rho\|y\|^{-1} y \in \rho B_{Y} \cap$ $R(Z)$. It follows that there exists $x \in B_{X} \cap D(Z)$ such that $\rho\|y\|^{-1} y \in Z(x)$, so that $y \in Z\left(\rho^{-1}\|y\| x\right)$. Hence,

$$
\left(\left(\rho^{-1}\|y\| x, y\right), 0\right) \in Z \times M(Z)
$$

and

$$
\left\|\left(\left(\rho^{-1}\|y\| x, y\right), 0\right)\right\| \leq k\|y\|,
$$

where $k=\rho^{-1}+1$. Note that

$$
j_{Z}\left(\left(\rho^{-1}\|y\| x, y\right), 0\right)=y .
$$

Hence, if $\|y\| \leq k^{-1}$ then $\left\|\left(\left(\rho^{-1}\|y\| x, y\right), 0\right)\right\| \leq 1$. Consequently,

$$
k^{-1} B_{Y} \cap R\left(j_{Z}\right) \subset j_{Z}\left(B_{Z \times M(Z)}\right)
$$

that is, $j_{Z}$ is open.

Conversely, assume that there exists $\rho>0$ such that

$$
\rho B_{Y} \cap R\left(j_{Z}\right) \subset j_{Z}\left(B_{Z \times M(Z)}\right) .
$$

Let $y \in R(Z)=R\left(j_{Z}\right)$ be such that $\|y\| \leq \rho$. It follows that there exists $\left(\left(x, y_{1}\right), y_{0}\right) \in$ $B_{Z \times M(Z)}$ (in particular, $x \in B_{X} \cap D(Z)$ ) such that

$$
j_{Z}\left(\left(x, y_{1}\right), y_{0}\right)=y_{1}-y_{0}=y \text {. }
$$

Since $\left(x, y_{1}\right) \in Z$ and $\left(0, y_{0}\right) \in Z$, then $\left(x, y_{1}-y_{0}\right) \in Z$ and $y \in Z(x)$. Consequently, (7) holds, so the relation $Z$ is open.

Now, consider a sequence $\left\{\left(\left(0, y_{n}\right), y_{n}\right)\right\} \subset R\left(i_{Z}\right)$ converging to $\left((x, y), y_{0}\right)$ such that $\left((x, y), y_{0}\right) \in Z \times M(Z)$. It follows that $x=0, y=y_{0}$ and because $y_{0} \in M(Z)$, we deduce that $\left((x, y), y_{0}\right)=\left(\left(0, y_{0}\right), y_{0}\right) \in R\left(i_{Z}\right)$. This implies that $R\left(i_{Z}\right)$ is closed in $Z \times M(Z)$.

In the next lemma we compute the adjoints of $i_{Z}$ and $j_{Z}$. 
LEMMA 9. Let $X, Y$ be two normed spaces, $Z \subset X \times Y$ be a linear relation and $i_{Z}, j_{Z}$ be the bounded operators associated with $Z$. Then $i_{Z}^{\prime}: Z^{\prime} \times M(Z)^{\prime} \rightarrow M(Z)^{\prime}$ is given by

$$
i_{Z}^{\prime}\left(z^{\prime}, y_{0}^{\prime}\right)=z^{\prime} \circ h_{1}+y_{0}^{\prime}, \quad \forall\left(z^{\prime}, y_{0}^{\prime}\right) \in Z^{\prime} \times M(Z)^{\prime},
$$

where

$$
h_{1}: M(Z) \rightarrow\{0\} \times M(Z), \quad h_{1}\left(y_{0}\right)=\left(0, y_{0}\right)
$$

and $j_{Z}^{\prime}: Y^{\prime} \rightarrow Z^{\prime} \times M(Z)^{\prime}$ is given by

$$
j_{Z}^{\prime}\left(y^{\prime}\right)=\left(\left(0, y^{\prime}\right)\left|Z,-y^{\prime}\right| M(Z)\right), \quad \forall y^{\prime} \in Y^{\prime} .
$$

Proof. Consider $y_{0} \in M(Z)$ and $\left(z^{\prime}, y_{0}^{\prime}\right) \in Z^{\prime} \times M(Z)^{\prime}$. Then

$$
\begin{aligned}
\left\langle y_{0}, i_{Z}^{\prime}\left(z^{\prime}, y_{0}^{\prime}\right)\right\rangle & =\left\langle i_{Z}\left(y_{0}\right),\left(z^{\prime}, y_{0}^{\prime}\right)\right\rangle \\
& =\left\langle\left(\left(0, y_{0}\right), y_{0}\right),\left(z^{\prime}, y_{0}^{\prime}\right)\right\rangle \\
& =z^{\prime}\left(0, y_{0}\right)+y_{0}^{\prime}\left(y_{0}\right),
\end{aligned}
$$

which implies (8). On the other hand, consider $\left((x, y), y_{0}\right) \in Z \times M(Z)$ and $y^{\prime} \in Y^{\prime}$. Then

$$
\begin{aligned}
\left\langle\left((x, y), y_{0}\right), j_{Z}^{\prime}\left(y^{\prime}\right)\right\rangle & =\left\langle j_{Z}\left((x, y), y_{0}\right), y^{\prime}\right\rangle \\
& =\left\langle y-y_{0}, y^{\prime}\right\rangle \\
& =y^{\prime}(y)-y^{\prime}\left(y_{0}\right),
\end{aligned}
$$

which implies (9).

In Lemma 8 we have shown that there exists a very useful relation between $Z$ and a certain complex of normed spaces. In order to apply the theory of complexes of Banach spaces we consider the 'adjoint' complex and show in the next lemma that this is also related to $Z$.

LEMmA 10. Let $X, Y$ be two normed spaces and $Z \subset X \times Y$ be a linear relation. Consider the complex (6) associated with $Z$. Then the short sequence

$$
0 \rightarrow Y^{\prime} \stackrel{j_{Z}^{\prime}}{\rightarrow} Z^{\prime} \times M(Z)^{\prime} \stackrel{i_{Z}^{\prime}}{\rightarrow} M(Z)^{\prime} \rightarrow 0
$$

is a complex of Banach spaces satisfying

$$
\begin{aligned}
\operatorname{codim}\left(\overline{R\left(j_{Z}\right)}\right) & =\operatorname{dim}\left(N\left(j_{Z}^{\prime}\right)\right), \\
\operatorname{dim}\left(N\left(j_{Z}\right) / R\left(i_{Z}\right)\right) & \leq \operatorname{dim}\left(N\left(i_{Z}^{\prime}\right) / R\left(j_{Z}^{\prime}\right)\right),
\end{aligned}
$$

and

$$
\operatorname{dim}\left(N\left(i_{Z}\right)\right)=\operatorname{codim}\left(R\left(i_{Z}^{\prime}\right)\right)=0
$$


Moreover, if $Z$ is open then $R\left(j_{Z}^{\prime}\right)=N\left(j_{Z}\right)^{\perp}$,

$$
\operatorname{dim}\left(N\left(j_{Z}\right) / R\left(i_{Z}\right)\right)=\operatorname{dim}\left(N\left(i_{Z}^{\prime}\right) / R\left(j_{Z}^{\prime}\right)\right),
$$

and if $\operatorname{dim}\left(N\left(i_{Z}^{\prime}\right) / R\left(j_{Z}^{\prime}\right)\right)$ is finite and (11) holds, then $Z$ is open.

PROOF. First of all we remark that $i_{Z}^{\prime}$ is surjective, so from Lemma 8 we deduce that $\operatorname{dim}\left(N\left(i_{Z}\right)\right)=\operatorname{codim}\left(R\left(i_{Z}^{\prime}\right)\right)=0$. On the other hand, we know that $R\left(i_{Z}\right) \subset N\left(j_{Z}\right)$, which implies that $N\left(j_{Z}\right)^{\perp} \subset R\left(i_{Z}\right)^{\perp}$, which together with (see Lemma 7 (i))

$$
R\left(i_{Z}\right)^{\perp}=N\left(i_{Z}^{\prime}\right), \quad \overline{R\left(j_{Z}^{\prime}\right)} \subset N\left(j_{Z}\right)^{\perp}
$$

imply that

$$
R\left(j_{Z}^{\prime}\right) \subset N\left(i_{Z}^{\prime}\right),
$$

hence we have that (10) is a complex of Banach spaces. On the other hand, the equality $\operatorname{codim}\left(\overline{R\left(j_{Z}\right)}\right)=\operatorname{dim}\left(N\left(j_{Z}^{\prime}\right)\right)$ follows from Lemma 7(iii). Using (12), the fact that $R\left(i_{Z}\right)$ is closed in $Z \times M(Z)$ and Remark 6, we deduce that

$$
\operatorname{dim}\left(N\left(j_{Z}\right) / R\left(i_{Z}\right)\right)=\operatorname{dim}\left(R\left(i_{Z}\right)^{\perp} / N\left(j_{Z}\right)^{\perp}\right)=\operatorname{dim}\left(N\left(i_{Z}^{\prime}\right) / N\left(j_{Z}\right)^{\perp}\right) .
$$

From (13) we infer that

$$
N\left(i_{Z}^{\prime}\right) / N\left(j_{Z}\right)^{\perp} \simeq\left(N\left(i_{Z}^{\prime}\right) / R\left(j_{Z}^{\prime}\right)\right) /\left(N\left(j_{Z}\right)^{\perp} / R\left(j_{Z}^{\prime}\right)\right),
$$

which implies that

$$
\operatorname{dim}\left(N\left(i_{Z}^{\prime}\right) / N\left(j_{Z}\right)^{\perp}\right)+\operatorname{dim}\left(N\left(j_{Z}\right)^{\perp} / R\left(j_{Z}^{\prime}\right)\right)=\operatorname{dim}\left(N\left(i_{Z}^{\prime}\right) / R\left(j_{Z}^{\prime}\right)\right) .
$$

From (14) and (15) we deduce that

$$
\operatorname{dim}\left(N\left(j_{Z}\right) / R\left(i_{Z}\right)\right) \leq \operatorname{dim}\left(N\left(i_{Z}^{\prime}\right) / R\left(j_{Z}^{\prime}\right)\right) .
$$

If $Z$ is open, then using Lemma 8 it follows that $j_{Z}$ is open, hence applying Lemma 7(ii) we obtain that $R\left(j_{Z}^{\prime}\right)=N\left(j_{Z}\right)^{\perp}$, which together with (14) imply (11).

Now, assume that $\operatorname{dim}\left(N\left(i_{Z}^{\prime}\right) / R\left(j_{Z}^{\prime}\right)\right)$ is finite and that (11) holds. This together with (14) and (15) implies that $\operatorname{dim}\left(N\left(j_{Z}\right)^{\perp} / R\left(j_{Z}^{\prime}\right)\right)=0$, hence we obtain that $R\left(j_{Z}^{\prime}\right)=N\left(j_{Z}\right)^{\perp}$, and, again applying Lemma 7(ii), we deduce that $j_{Z}$ is open. Now the openness of $Z$ follows from Lemma 8 .

Proof of Theorem 1. First of all we introduce some isometric isomorphisms (see Lemma 5(i)) as follows:

$$
\begin{array}{r}
k:\{0\} \times Y^{\prime} \times\{0\} \rightarrow Y^{\prime}, \quad k\left(0, y^{\prime}, 0\right)=y^{\prime}, \\
l: M\left(Z_{1}\right)^{\prime} \rightarrow\{0\} \times\{0\} \times M\left(Z_{1}\right)^{\prime}, \quad l\left(y_{0}^{\prime}\right)=\left(0,0, y_{0}^{\prime}\right),
\end{array}
$$


and

$$
\varphi_{Z_{1}}:\left(X^{\prime} \times Y^{\prime} \times M\left(Z_{1}\right)^{\prime}\right) /\left(\left(Z_{1} \times M\left(Z_{1}\right)\right)^{\perp}\right) \rightarrow Z_{1}^{\prime} \times M\left(Z_{1}\right)^{\prime}
$$

defined by

$$
\varphi_{Z_{1}}\left(\left(x^{\prime}, y^{\prime}, y_{0}^{\prime}\right)+\left(Z_{1} \times M\left(Z_{1}\right)\right)^{\perp}\right)=\left(\left(x^{\prime}, y^{\prime}\right) \mid Z_{1}, y_{0}^{\prime}\right) .
$$

It follows that

$$
\varphi_{Z_{1}}^{-1}: Z_{1}^{\prime} \times M\left(Z_{1}\right)^{\prime} \rightarrow\left(X^{\prime} \times Y^{\prime} \times M\left(Z_{1}\right)^{\prime}\right) /\left(\left(Z_{1} \times M\left(Z_{1}\right)\right)^{\perp}\right)
$$

is given by

$$
\varphi_{Z_{1}}^{-1}\left(z^{\prime}, y_{0}^{\prime}\right)=\left(x^{\prime}, y^{\prime}, y_{0}^{\prime}\right)+\left(Z_{1} \times M\left(Z_{1}\right)\right)^{\perp}
$$

where $\left(x^{\prime}, y^{\prime}\right) \in X^{\prime} \times Y^{\prime}$ is such that $\left(x^{\prime}, y^{\prime}\right) \mid Z_{1}=z^{\prime}$.

Consider the operators

$$
\tilde{i}_{Z_{1}}=l \circ i_{Z_{1}}^{\prime} \circ \varphi_{Z_{1}}, \quad \tilde{j}_{Z_{1}}=\varphi_{Z_{1}}^{-1} \circ j_{Z_{1}}^{\prime} \circ k
$$

and the short sequence

$$
\begin{aligned}
& 0 \rightarrow\{0\} \times Y^{\prime} \times\{0\} \stackrel{\tilde{j}_{Z_{1}}}{\longrightarrow}\left(X^{\prime} \times Y^{\prime} \times M\left(Z_{1}\right)^{\prime}\right) /\left(\left(Z_{1} \times M\left(Z_{1}\right)\right)^{\perp}\right) \\
& \stackrel{\widetilde{i}_{Z_{1}}}{\longrightarrow}\{0\} \times\{0\} \times M\left(Z_{1}\right)^{\prime} \rightarrow 0 .
\end{aligned}
$$

Note that

$$
\varphi_{Z_{1}}\left(R\left(\tilde{j}_{Z_{1}}\right)\right)=R\left(j_{Z_{1}}^{\prime}\right), \quad \varphi_{Z_{1}}\left(N\left(\widetilde{i}_{Z_{1}}\right)\right)=N\left(i_{Z_{1}}^{\prime}\right),
$$

and

$$
k\left(N\left(\tilde{j}_{Z_{1}}\right)\right)=N\left(j_{Z_{1}}^{\prime}\right) .
$$

Using (1), Lemmas 5, 8, 10, Equations (17), $\underset{\sim}{(18)}$ and the fact that $Z_{1}$ is open, we deduce that (16) is a complex of Banach spaces, $\widetilde{i}_{Z_{1}}$ is surjective, $R\left(\widetilde{j}_{Z_{1}}\right)$ is closed and

$$
\begin{aligned}
\operatorname{dim}\left(N\left(\tilde{i}_{Z_{1}}\right) / R\left(\tilde{j}_{Z_{1}}\right)\right) & =\operatorname{dim}\left(N\left(Z_{1}\right)\right)<\infty, \\
\operatorname{dim}\left(N\left(\tilde{j}_{Z_{1}}\right)\right) & =\operatorname{codim}\left(\overline{R\left(Z_{1}\right)}\right)<\infty .
\end{aligned}
$$

It follows that the complex (16) is Fredholm and

$$
-\overline{\mathrm{ind}}\left(Z_{1}\right)=\operatorname{ind}((16)) \text {. }
$$

Now, taking into account that $M\left(Z_{1}\right)=M\left(Z_{2}\right)$ and replacing $Z_{1}$ by $Z_{2}$, we associate with $Z_{2}$ the complex of Banach spaces

$$
\begin{aligned}
0 \rightarrow\{0\} \times Y^{\prime} \times\{0\} \stackrel{\tilde{j}_{Z_{2}}}{\longrightarrow}\left(X^{\prime} \times Y^{\prime} \times M\left(Z_{1}\right)^{\prime}\right) /\left(\left(Z_{2} \times M\left(Z_{1}\right)\right)^{\perp}\right) \\
\stackrel{\tilde{i}_{Z_{2}}}{\longrightarrow}\{0\} \times\{0\} \times M\left(Z_{1}\right)^{\prime} \rightarrow 0 .
\end{aligned}
$$


We now characterize $G_{0}\left(\tilde{j}_{Z_{j}}\right)$ for $j=1,2$. Consider

$$
\left(\left(0, y^{\prime}, 0\right),\left(u^{\prime}, v^{\prime}, y_{0}^{\prime}\right)\right) \in G_{0}\left(\tilde{j}_{Z_{j}}\right)
$$

It follows that

$$
\widetilde{j}_{Z_{j}}\left(0, y^{\prime}, 0\right)=\left(u^{\prime}, v^{\prime}, y_{0}^{\prime}\right)+\left(Z_{j} \times M\left(Z_{1}\right)\right)^{\perp} .
$$

On the other hand, from Lemma 9,

$$
\widetilde{j}_{Z_{j}}\left(0, y^{\prime}, 0\right)=\left(0, y^{\prime},-y^{\prime} \mid M\left(Z_{1}\right)\right)+\left(Z_{j} \times M\left(Z_{1}\right)\right)^{\perp} .
$$

It follows that

$$
\left(0, y^{\prime},-y^{\prime} \mid M\left(Z_{1}\right)\right)\left|\left[Z_{j} \times M\left(Z_{1}\right)\right]=\left(u^{\prime}, v^{\prime}, y_{0}^{\prime}\right)\right|\left[Z_{j} \times M\left(Z_{1}\right)\right],
$$

that is,

$$
y^{\prime}(y)-y^{\prime}\left(y_{0}\right)=u^{\prime}(x)+v^{\prime}(y)+y_{0}^{\prime}\left(y_{0}\right), \quad \forall\left((x, y), y_{0}\right) \in Z_{j} \times M\left(Z_{1}\right) .
$$

Taking in the above relation $\left((x, y), y_{0}\right)=\left((0,0), y_{0}\right)$, it follows that

$$
-y^{\prime}\left(y_{0}\right)=y_{0}^{\prime}\left(y_{0}\right), \quad \forall y_{0} \in M\left(Z_{1}\right),
$$

and

$$
y^{\prime}(y)=u^{\prime}(x)+v^{\prime}(y), \quad \forall(x, y) \in Z_{j}
$$

We deduce that

$$
\left(\left(0, y^{\prime}, 0\right),\left(u^{\prime}, v^{\prime}, y_{0}^{\prime}\right)\right)=\left(\left(0, y^{\prime}, 0\right),\left(u^{\prime}, v^{\prime},-y^{\prime} \mid M\left(Z_{1}\right)\right)\right)
$$

where

$$
\left(u^{\prime}, v^{\prime}\right)\left|Z_{j}=\left(0, y^{\prime}\right)\right| Z_{j}
$$

On the other hand, arguing as above,

$$
\left(\left(0, y^{\prime}, 0\right),\left(u^{\prime}, v^{\prime},-y^{\prime} \mid M\left(Z_{1}\right)\right)\right) \in G_{0}\left(\tilde{j}_{Z_{j}}\right)
$$

for all $u^{\prime} \in X^{\prime}$ and $y^{\prime}, v^{\prime} \in Y^{\prime}$ such that (22) is satisfied. Further, we characterize $G_{0}\left(\widetilde{i}_{Z_{j}}\right) \quad(j=1,2)$. Actually, from Lemma 9 it follows immediately that $\left(\left(x^{\prime}, y^{\prime}, y_{0}^{\prime}\right),\left(0,0, y_{1}^{\prime}\right)\right) \in G_{0}\left(\widetilde{i}_{Z_{j}}\right)$ if and only if $y^{\prime} \mid M\left(Z_{1}\right)+y_{0}^{\prime}=y_{1}^{\prime}$. Therefore,

$$
G_{0}\left(\tilde{i}_{Z_{1}}\right)=G_{0}\left(\tilde{i}_{Z_{2}}\right)
$$

Next we prove that

$$
\delta\left(G_{0}\left(\tilde{j}_{Z_{1}}\right), G_{0}\left(\tilde{j}_{Z_{2}}\right)\right) \leq \delta\left(Z_{1}^{\perp}, Z_{2}^{\perp}\right)
$$


that is,

$$
\sup _{s_{1} \in G_{0}\left(\tilde{j}_{Z_{1}}\right),\left\|s_{1}\right\| \leq 1} \inf _{s_{2} \in G_{0}\left(\widetilde{j}_{Z_{2}}\right)}\left\|s_{1}-s_{2}\right\| \leq \sup _{t_{1} \in Z_{1}^{\perp},\left\|t_{1}\right\| \leq 1} \inf _{t_{2} \in Z_{2}^{\perp}}\left\|t_{1}-t_{2}\right\| .
$$

Let $s_{1} \in G_{0}\left(\tilde{j}_{Z_{1}}\right)$ be such that $\left\|s_{1}\right\| \leq 1$. Using the above characterization of $G_{0}\left(\tilde{j}_{Z_{1}}\right)$, it follows that

$$
s_{1}=\left(\left(0, y^{\prime}, 0\right),\left(u_{1}^{\prime}, v_{1}^{\prime},-y^{\prime} \mid M\left(Z_{1}\right)\right)\right)
$$

where $u_{1}^{\prime} \in X^{\prime}, y^{\prime}, v_{1}^{\prime} \in Y^{\prime}$ and $\left(u_{1}^{\prime}, v_{1}^{\prime}\right)\left|Z_{1}=\left(0, y^{\prime}\right)\right| Z_{1}$. Hence, taking

$$
t_{1}=\left(u_{1}^{\prime}, v_{1}^{\prime}\right)-\left(0, y^{\prime}\right) \text {, }
$$

$t_{1} \in Z_{1}^{\perp}$ and $\left\|t_{1}\right\| \leq 1$. Let $t_{2} \in Z_{2}^{\perp}$ be fixed and consider

$$
\left(u_{2}^{\prime}, v_{2}^{\prime}\right)=t_{2}+\left(0, y^{\prime}\right) \text {. }
$$

It follows that

$$
s_{2}:=\left(\left(0, y^{\prime}, 0\right),\left(u_{2}^{\prime}, v_{2}^{\prime},-y^{\prime} \mid M\left(Z_{1}\right)\right)\right) \in G_{0}\left(\tilde{j}_{Z_{2}}\right)
$$

and $\left\|s_{1}-s_{2}\right\|=\left\|t_{1}-t_{2}\right\|$. Consequently, (24) holds. Interchanging $Z_{1}$ and $Z_{2}$, it follows that

$$
\widehat{\delta}\left(G_{0}\left(\tilde{j}_{Z_{1}}\right), G_{0}\left(\tilde{j}_{Z_{2}}\right)\right) \leq \widehat{\delta}\left(Z_{1}^{\perp}, Z_{2}^{\perp}\right),
$$

which together with Lemma 5 (iii) imply that

$$
\widehat{\delta}\left(G_{0}\left(\widetilde{j}_{Z_{1}}\right), G_{0}\left(\widetilde{j}_{Z_{2}}\right)\right) \leq \widehat{\delta}\left(\overline{Z_{1}}, \overline{Z_{2}}\right) .
$$

From (23) and (25) we deduce that

$$
\widehat{\delta}_{0}((16),(21)) \leq \widehat{\delta}\left(\overline{Z_{1}}, \overline{Z_{2}}\right) .
$$

Hence, using Theorem 4 , it follows that there exists $\varepsilon>0$ such that if $\widehat{\delta}\left(\overline{Z_{1}}, \overline{Z_{2}}\right)<\varepsilon$, then the complex (21) is Fredholm,

$$
\operatorname{dim}\left(N\left(\tilde{j}_{Z_{2}}\right)\right) \leq \operatorname{dim}\left(N\left(\tilde{j}_{Z_{1}}\right)\right), \quad \operatorname{codim}\left(R\left(\widetilde{i}_{Z_{2}}\right)\right)=\operatorname{codim}\left(R\left(\tilde{i}_{Z_{1}}\right)\right)=0,
$$

and

$$
\operatorname{dim}\left(N\left(\widetilde{i}_{Z_{2}}\right) / R\left(\tilde{j}_{Z_{2}}\right)\right) \leq \operatorname{dim}\left(N\left(\tilde{i}_{Z_{1}}\right) / R\left(\tilde{j}_{Z_{1}}\right)\right)
$$

Moreover,

$$
\operatorname{ind}((16))=\operatorname{ind}((21)) \text {. }
$$

From Lemmas 8 and 10 it follows that

$$
\operatorname{dim}\left(N\left(Z_{2}\right)\right) \leq \operatorname{dim}\left(N\left(\tilde{i}_{Z_{2}}\right) / R\left(\tilde{j}_{Z_{2}}\right)\right), \quad \operatorname{codim}\left(\overline{R\left(Z_{2}\right)}\right)=\operatorname{dim}\left(N\left(\tilde{j}_{Z_{2}}\right)\right) .
$$


Using (29), (27) and (19) it follows that

$$
\operatorname{dim}\left(N\left(Z_{2}\right)\right) \leq \operatorname{dim}\left(N\left(\tilde{i}_{Z_{2}}\right) / R\left(\tilde{j}_{Z_{2}}\right)\right) \leq \operatorname{dim}\left(N\left(\tilde{i}_{Z_{1}}\right) / R\left(\tilde{j}_{Z_{1}}\right)\right)=\operatorname{dim}\left(N\left(Z_{1}\right)\right),
$$

and using (29), (26) and (19) it follows that

$$
\operatorname{codim}\left(\overline{R\left(Z_{2}\right)}\right)=\operatorname{dim}\left(N\left(\tilde{j}_{Z_{2}}\right)\right) \leq \operatorname{dim}\left(N\left(\tilde{j}_{Z_{1}}\right)\right)=\operatorname{codim}\left(\overline{R\left(Z_{1}\right)}\right),
$$

hence (2) holds. On the other hand, from (29), (26), (28) and (20) we deduce that

$$
\begin{aligned}
\overline{\operatorname{ind}}\left(Z_{2}\right) & =\operatorname{dim}\left(N\left(Z_{2}\right)\right)-\operatorname{codim}\left(\overline{R\left(Z_{2}\right)}\right) \\
& \left.\leq \operatorname{dim}\left(N\left(\widetilde{i}_{Z_{2}}\right) / R\left(\tilde{j}_{Z_{2}}\right)\right)\right)-\operatorname{dim}\left(N\left(\widetilde{j}_{Z_{2}}\right)\right) \\
& =-\operatorname{ind}((21))=-\operatorname{ind}((16))=\overline{\operatorname{ind}}\left(Z_{1}\right),
\end{aligned}
$$

hence (3) holds.

We prove now the last assertion of the theorem. Let us suppose that $\overline{\operatorname{ind}}\left(Z_{2}\right)=$ $\overline{\operatorname{ind}}\left(Z_{1}\right)$. From (30) we infer that

$$
\operatorname{dim}\left(N\left(Z_{2}\right)\right)-\operatorname{codim}\left(\overline{R\left(Z_{2}\right)}\right)=\operatorname{dim}\left(N\left(i_{Z_{2}}^{\prime}\right) / R\left(j_{Z_{2}}^{\prime}\right)\right)-\operatorname{dim}\left(N\left(j_{Z_{2}}^{\prime}\right)\right) .
$$

On the other hand, from Lemma 10 we obtain that

$$
\operatorname{codim}\left(\overline{R\left(Z_{2}\right)}\right)=\operatorname{dim}\left(N\left(j_{Z_{2}}^{\prime}\right)\right)
$$

and from Lemma 8

$$
\operatorname{dim}\left(N\left(Z_{2}\right)\right)=\operatorname{dim}\left(N\left(j_{Z_{2}}\right) / R\left(i_{Z_{2}}\right)\right) .
$$

Introduced in (31), relations (32) and (33) imply that

$$
\operatorname{dim}\left(N\left(j_{Z_{2}}\right) / R\left(i_{Z_{2}}\right)\right)=\operatorname{dim}\left(N\left(i_{Z_{2}}^{\prime}\right) / R\left(j_{Z_{2}}^{\prime}\right)\right) .
$$

Using Lemma 10 again, we deduce that $Z_{2}$ is open. Finally, similar arguments show that condition $\overline{\operatorname{ind}}\left(Z_{2}\right)=\overline{\operatorname{ind}}\left(Z_{1}\right)$ is also necessary to obtain that $Z_{2}$ is open. The proof is now complete.

REMARK 11. (i) In fact, in (24) we can prove that

$$
\delta\left(G_{0}\left(\tilde{j}_{Z_{1}}\right), G_{0}\left(\tilde{j}_{Z_{2}}\right)\right)=\delta\left(Z_{1}^{\perp}, Z_{2}^{\perp}\right) .
$$

Indeed, let us consider $t_{1} \in Z_{1}^{\perp}$ such that $\left\|t_{1}\right\| \leq 1$ and define $s_{1} \in G_{0}\left(\tilde{j} Z_{1}\right)$ with $\left\|s_{1}\right\| \leq 1$ by

$$
s_{1}=\left((0,0,0),\left(u_{1}^{\prime}, v_{1}^{\prime}, 0\right)\right) \quad \text { where }\left(u_{1}^{\prime}, v_{1}^{\prime}\right):=t_{1} \text {. }
$$

Let

$$
s_{2}=\left(\left(0, y^{\prime}, 0\right),\left(u_{2}^{\prime}, v_{2}^{\prime},-y^{\prime} \mid M\left(Z_{1}\right)\right)\right) \in G_{0}\left(\tilde{j}_{Z_{2}}\right)
$$


be fixed and $t_{2} \in Z_{2}^{\perp}$ given by $t_{2}=\left(u_{2}^{\prime}, v_{2}^{\prime}\right)-\left(0, y^{\prime}\right)$. Then

$$
\begin{aligned}
\left\|s_{1}-s_{2}\right\| & =\left\|y^{\prime}\right\|+\left\|u_{1}^{\prime}-u_{2}^{\prime}\right\|+\left\|v_{1}^{\prime}-v_{2}^{\prime}\right\|+\left\|y^{\prime} \mid M\left(Z_{1}\right)\right\|, \\
\left\|t_{1}-t_{2}\right\| & =\left\|u_{1}^{\prime}-u_{2}^{\prime}\right\|+\left\|v_{1}^{\prime}-v_{2}^{\prime}+y^{\prime}\right\| \leq\left\|u_{1}^{\prime}-u_{2}^{\prime}\right\|+\left\|v_{1}^{\prime}-v_{2}^{\prime}\right\|+\left\|y^{\prime}\right\|,
\end{aligned}
$$

hence $\left\|t_{1}-t_{2}\right\| \leq\left\|s_{1}-s_{2}\right\|$. Our claim is now proved.

Hence, interchanging $Z_{1}$ and $Z_{2}$ and applying Lemma 5(iii), it follows that

$$
\widehat{\delta}_{0}((16),(21))=\widehat{\delta}\left(\overline{Z_{1}}, \overline{Z_{2}}\right),
$$

so, we can apply Albrecht-Vasilescu theorem to the complexes (16) and (21) if and only if $\widehat{\delta}\left(\overline{Z_{1}}, \overline{Z_{2}}\right)$ is sufficiently small.

(ii) Let $Z_{1} \subset X \times Y$ be a linear relation, and let $A \in \mathcal{B}(D(A), Y)$ be such that $D\left(Z_{1}\right) \subset D(A)$. Consider $Z_{2}=Z_{1}+A$. It is clear that $M\left(Z_{1}\right)=M\left(Z_{2}\right)$. On the other hand, one can show that

$$
\widehat{\delta}\left(G_{0}\left(\tilde{j}_{Z_{1}}\right), G_{0}\left(\tilde{j}_{Z_{2}}\right)\right) \leq\|A\| .
$$

Indeed, let $s_{1} \in G_{0}\left(\tilde{j}_{Z_{1}}\right)$ be such that $\left\|s_{1}\right\| \leq 1$. Using the characterization of $G_{0}\left(\tilde{j}_{Z_{1}}\right)$, it follows that $s_{1}=\left(\left(0, y^{\prime}, 0\right),\left(u_{1}^{\prime}, v_{1}^{\prime},-y^{\prime} \mid M\left(Z_{1}\right)\right)\right)$, where $u_{1}^{\prime} \in X^{\prime}, y^{\prime}, v_{1}^{\prime} \in Y^{\prime}$ and $\left(u_{1}^{\prime}, v_{1}^{\prime}\right)\left|Z_{1}=\left(0, y^{\prime}\right)\right| Z_{1}$. Let us introduce the notation

$$
\xi_{1}=\left(u_{1}^{\prime}, v_{1}^{\prime}\right) \in X^{\prime} \times Y^{\prime}, \quad \gamma=\left(0, y^{\prime}\right) \in X^{\prime} \times Y^{\prime} .
$$

Then

$$
\xi_{1}\left|Z_{1}=\gamma\right| Z_{1}
$$

Consider

$$
\lambda=\gamma \mid Z_{2} \in Z_{2}^{\prime}
$$

For any $(x, y) \in Z_{1}$,

$$
\left|\xi_{1}(x, y+A x)-\lambda(x, y+A x)\right|=\left|v_{1}^{\prime}(A x)-y^{\prime}(A x)\right| \leq\left\|v_{1}^{\prime}-y^{\prime}\right\|\|A\|\|x\|,
$$

and because $\|x\| \leq\|(x, y+A x)\|$ and $\left\|v_{1}^{\prime}-y^{\prime}\right\| \leq\left\|v_{1}^{\prime}\right\|+\left\|y^{\prime}\right\| \leq 1$, it follows that

$$
\left\|\xi_{1} \mid Z_{2}-\lambda\right\| \leq\|A\| \text {. }
$$

Using the Hahn-Banach theorem, we deduce that there exists $\tilde{\xi}_{1} \in X^{\prime} \times Y^{\prime}$ such that $\widetilde{\xi}_{1}\left|Z_{2}=\xi_{1}\right| Z_{2}-\lambda$ and $\left\|\widetilde{\xi}_{1}\right\|=\left\|\xi_{1} \mid Z_{2}-\lambda\right\| \leq\|A\|$. Consider $\xi_{2}=\left(u_{2}^{\prime}, v_{2}^{\prime}\right) \in X^{\prime} \times Y^{\prime}$ given by $\xi_{2}=\xi_{1}-\widetilde{\xi}_{1}$. It follows that

$$
\left(u_{2}^{\prime}, v_{2}^{\prime}\right)\left|Z_{2}=\left(0, y^{\prime}\right)\right| Z_{2},
$$

hence

$$
s_{2}=\left(\left(0, y^{\prime}, 0\right),\left(u_{2}^{\prime}, v_{2}^{\prime},-y^{\prime} \mid M\left(Z_{1}\right)\right)\right) \in G_{0}\left(\tilde{j}_{Z_{2}}\right)
$$


and

$$
\left\|s_{1}-s_{2}\right\|=\left\|\widetilde{\xi}_{1}\right\| \leq\|A\|
$$

Hence, $\delta\left(G_{0}\left(\tilde{j}_{Z_{1}}\right), G_{0}\left(\tilde{j}_{Z_{2}}\right)\right) \leq\|A\|$. Replacing $Z_{1}$ by $Z_{2}, A$ by $-A$ and using the equality $Z_{2}-A=Z_{1}$, we deduce that $\delta\left(G_{0}\left(\widetilde{j}_{Z_{2}}\right), G_{0}\left(\widetilde{j}_{Z_{1}}\right)\right) \leq\|A\|$. Hence, our claim is proved.

(iii) Taking (ii) into account, the proof of Corollary 3 is similar to that of Theorem 1.

REMARK 12. In Theorem 1, if we replace condition

$$
\operatorname{codim}\left(\overline{R\left(Z_{1}\right)}\right)<\infty
$$

by

$$
\operatorname{codim}\left(\overline{R\left(Z_{1}\right)}\right)=\infty
$$

from the proof of Theorem 1 and from the Albrecht-Vasilescu theorem in the semiFredholm situation (see [1, Theorem 1.4], [6, Theorem 2.7]), it follows that

$$
\operatorname{codim}\left(\overline{R\left(Z_{2}\right)}\right)=\infty, \quad \operatorname{dim}\left(N\left(Z_{2}\right)\right) \leq \operatorname{dim}\left(N\left(Z_{1}\right)\right) .
$$

In particular,

$$
\overline{\operatorname{ind}}\left(Z_{2}\right)=\overline{\operatorname{ind}}\left(Z_{1}\right)=-\infty \text {. }
$$

On the other hand, if we replace condition

$$
\operatorname{dim}\left(N\left(Z_{1}\right)\right)<\infty
$$

by

$$
\operatorname{dim}\left(N\left(Z_{1}\right)\right)=\infty
$$

from the proof of Theorem 1 and from the Albrecht-Vasilescu theorem in the semiFredholm case it follows that

$$
\operatorname{dim}\left(N\left(Z_{2}\right)\right) \leq \operatorname{dim}\left(N\left(Z_{1}\right)\right)
$$

(with equality if $Z_{2}$ is open) and

$$
\operatorname{codim}\left(\overline{R\left(Z_{2}\right)}\right) \leq \operatorname{codim}\left(\overline{R\left(Z_{1}\right)}\right) .
$$

\section{References}

[1] E. Albrecht and F.-H. Vasilescu, 'Stability of the index of a semi-Fredholm complex of Banach spaces', J. Funct. Anal. 66 (1986), 141-172.

[2] T. Alvarez, 'On almost semi-Fredholm linear relations in normed spaces', Glasgow Math. J. 47 (2005), 187-193. 
[3] _ Characterisations of open multivalued linear operators', Studia Math. 175 (2006), $205-212$.

[4] T. Alvarez, R. W. Cross and D. Wilcox, 'Multivalued Fredholm type operators with abstract generalised inverses', J. Math. Anal. Appl. 261 (2001), 403-417.

[5] T. Alvarez and D. Wilcox, 'Perturbation theory of multivalued Atkinson operators in normed spaces', Bull. Austral. Math. Soc. 76 (2007), 195-204.

[6] C.-G. Ambrozie and F.-H. Vasilescu, Banach Space Complexes, Mathematics and its Applications, 334 (Kluwer Academic, Dordrecht, 1995).

[7] R. Arens, 'Operational calculus of linear relations', Pacific J. Math. 11 (1961), 9-23.

[8] E. A. Coddington, Multivalued Operators and Boundary Value Problems, Lecture Notes in Mathematics, 183 (Springer, Berlin, 1971), pp. 2-8.

[9] R. W. Cross, Multivalued Linear Operators (Marcel Dekker, New York, 1998).

[10] A. Favini and A. Yagi, 'Multivalued linear operator and dengenerate evolution equations', Ann. Mat. Pura Appl. 163 (1993), 353-384.

[11] R. Harte, Invertibility and Singularity for Bounded Linear Operators (Marcel Dekker, New York, 1988).

[12] T. Kato, Perturbation Theory for Linear Operators (Springer, Berlin, 1966), pp. 233-304; Lecture Notes in Pure and Appl. Math., 83, Dekker, New York, 1983.

[13] F.-H. Vasilescu, 'Spectral theory in quotient Frechét spaces. I', Rev. Roumaine Math. Pures Appl. 32 (1987), 561-579.

[14] _ _ 'Spectral theory in quotient Frechét spaces. II', J. Operator Theory 21 (1989), 145-202.

DANA GHEORGHE, Department of Mathematics, University of Piteşti,

110040 Piteşti, Romania

e-mail: gheorghedana@yahoo.com 